\title{
BMJ Open Family-focused practices in addictions: a scoping review protocol
}

\author{
Toula Kourgiantakis, Rachelle Ashcroft
}

To cite: Kourgiantakis T, Ashcroft R. Familyfocused practices in addictions: a scoping review protocol. BMJ Open 2018;8:e019433. doi:10.1136/ bmjopen-2017-019433

- Prepublication history for this paper is available online. To view these files, please visit the journal online (http://dx.doi. org/10.1136/bmjopen-2017019433).

Received 1 September 2017 Revised 7 December 2017 Accepted 14 December 2017

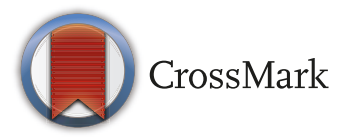

Factor-Inwentash Faculty of Social Work, University of Toronto, Toronto, Ontario, Canada

Correspondence to Dr Toula Kourgiantakis; toula.kourgiantakis@utoronto.ca

\begin{abstract}
Introduction Families are significantly impacted by addictions and family involvement in treatment can reduce the harms and can also improve treatment entry, treatment completion and treatment outcomes for the individual coping with an addiction. Although the benefits of familyfocused practices in addictions have been documented, services continue to have an individual focus and research on this topic is also limited. The objective of this study is to map the extent, range and nature of evidence available examining family interventions in addictions and identify gaps to guide future research, policy and practice. Methods and analysis This is a scoping review using the five-stage framework developed by Arksey and 0'Malley. We will include published and unpublished empirical studies focusing on any type of family interventions in addiction treatment between 2000 and the present in English or French. A reviewer will search for literature that meets the inclusion criteria through the following electronic databases: MEDLINE, PsycINFO and Social Services Abstracts. For a comprehensive search, we will also hand-search reference lists, web sites and key journals. Data will be charted and sorted using a thematic analysis approach.

Ethics and dissemination This review will be the first to examine all forms of family-focused practices for both substance use and problem gambling treatment for adults. It will provide information about existing service provisions and gaps in practice. This review can be used to start moving towards the development of best practices for families in addiction treatment. The results will be disseminated through a peer-reviewed journal and at mental health and addiction conferences.
\end{abstract}

\section{INTRODUCTION}

There are more than 29.5 million individuals worldwide who have a drug use problem ${ }^{1}$ and 76 million people with alcohol use disorder. ${ }^{2}$ More recently, there has been more research on behavioural addictions and studies show that problem gambling affects $0.12 \%-5.8 \%$ of people across five continents. ${ }^{3}$ Substance use disorders and problem gambling are linked with multifarious consequences including health concerns, ${ }^{4}$ financial difficulties, ${ }^{5}{ }^{6}$ stigma, discrimination and isolation. ${ }^{7}$ It is estimated that more than $50 \%$ of those seeking services for an addiction are also coping with mental illness, ${ }^{8}$ and many studies have

\section{Strengths and limitations of this study}

- This scoping review will be the first to examine family-focused practices in both substance use and gambling adult treatment.

- Inclusion criteria are broad (English and French, peer-reviewed and grey literature) and this will give the review breadth and comprehensiveness.

- The studies included in the review will not be appraised for quality.

- Rigour will be enhanced through the use of a data charting form and a transparent, iterative team approach.

also found that the rates of suicidal ideation, suicide attempts and completed suicides are elevated for individuals with an addiction..$^{9-11}$ There is also an increasing awareness of the impact of addictions on individuals and on families with more than 100 million family members affected by a relative's addiction. ${ }^{12}$ The addiction of a family member has many adverse effects for families such as high levels of distress, health problems, ${ }^{513}$ family conflict, domestic violence, child maltreatment ${ }^{12}$ and financial precarity. ${ }^{5612}$ Studies also report that the health costs for family members coping with the addiction of a significant other are considerably higher than family members who do not have a family member with an addiction. ${ }^{56}$ Despite the negative impact on families, addiction treatment has historically focused on the individual. ${ }^{14}$ Csiernik ${ }^{16}$ described family-focused services as the "neglected aspect of addiction treatment' and other researchers have stated that service providers view family members as 'adjuncts' and they are not perceived as an integral part of addiction treatment. ${ }^{17}$ This creates a significant barrier to family involvement in addictions. ${ }^{15} 1718$

The involvement of family members in routine addiction treatment has been documented as important for two important and inter-related reasons. First, as mentioned previously, families are negatively affected and family-focused services can help reduce the harm to individual family members and 
to the entire family unit. ${ }^{19} \mathrm{~A}$ second compelling reason for involving families in addiction treatment is that family involvement increases treatment entry, enhances treatment completion and is also linked with better treatment outcomes for the individual coping with the addiction. ${ }^{20-22}$ This is relevant when considering the fact that only $20 \%$ (or fewer) of individuals with substance use disorders seek treatment, ${ }^{23}$ and among problem gambling individuals only $3 \%-6 \%$ seek professional treatment. ${ }^{24}$ Among those who receive treatment, approximately $50 \%$ drop out of treatment and treatment completion is one of the factors most closely associated with positive treatment outcomes. ${ }^{25}{ }^{26}$ Although studies have documented robust evidence when involving families in addiction treatment, most services target the individual with the addiction and there is little evidence of family-focused approaches in addiction service provisions. ${ }^{14}$

Addiction researchers have identified three categories of family interventions: (1) working with family members to promote the entry and engagement in treatment of the individual with the addiction; (2) involving family members in the treatment of the individual with the addiction; and (3) providing services to family members in their own right. ${ }^{15} 19$ There have been a number of substance use studies on the first two categories. For the first category, a few studies have evaluated the Community Reinforcement Approach and Family Training (CRAFT) intervention. ${ }^{27-29}$ The CRAFT programme teaches family members coping skills and also facilitates treatment entry for the individual with addiction-related concerns. ${ }^{29}$ The second category of family interventions has also been studied, but it focuses on conjoint treatment and it has largely targeted spouses (eg, Alcohol Behaviour Couple Therapy).$^{30}$ The third category concentrates on services given directly to families, and it has received the least attention. While there have been scant studies on this third category of family interventions, a group of UK researchers have developed the stress-straincoping-support (SSCS) model aimed at reducing the stress and strain to families and increasing support and coping skills. ${ }^{31}$ This approach has been evaluated in a primary care setting and shows promising results. ${ }^{13}$ The SSCS model has similarities to the family psychoeducation approach-an evidence-based practice in mental health and a commonly used family approach for schizophrenia. ${ }^{32}$ The family psychoeducation literature states that all interventions for families should include the 'Big Three', a term that refers to information about the addiction/mental illness, coping skill development and support from peers and professionals. Despite the fact that there has been some research (notably in the first two categories), this is still limited even though the benefits of family involvement and the adverse effects of addictions on families have been documented. In light of the low rates of treatment seeking and high rates of attrition from treatment for individuals with addictions, as well as the pervasive adverse effects of addictions on families, it is critical that family-focused services are available and accessible for all families. The robust research in mental health has demonstrated that the 'Big Three' (psychoeducation, support and coping skills) is an evidence-based practice. ${ }^{32}$ The SSCS model has similar tenets and practices, but requires more research and implementation in policy and practice. Researchers have attributed this neglect to 'a consequence of the lack of a family orientation in professional training and practice, plus the existence of a number of models of family functioning that cast family members in a negative light. ${ }^{, 33}$ To our knowledge, there have not been any reviews on family involvement in both substance use and problem gambling treatment for adults. ${ }^{34}$ Disordered gambling is the only behavioural addiction in the Diagnostic and Statistical Manual of Mental Disorders, Fifth Edition, and it is classified with substance use disorders due to their similarities in symptomatology. ${ }^{35}$ This scoping review will focus on all three forms of family involvement in substance use and gambling treatment to elucidate our understanding of a range of programmes that promote family-focused treatment. It will also identify any gaps in service provisions, as well as barriers to implementation of family-focused practices that have been identified in the literature. This will be an important guide to help move towards best practices for families in addiction treatment. There are currently no clearly identified best practices and this scoping review can be used by both policymakers and clinical settings to develop policies and implement best practices in addiction treatment with various family-focused interventions.

\section{STUDY OBJECTIVES}

The objectives for this scoping review on family involvement and interventions in addictions are as follows:

- Map and categorise the extent, range and nature of evidence available in peer-reviewed and grey literature examining family interventions in substance use disorders and problem gambling.

- Identify recent family interventions in adult addiction treatment programmes, as well as treatment gaps to guide future research, policy development and service provisions.

\section{METHODS AND ANALYSIS}

We will conduct a scoping review to examine the literature on family involvement in addictions using a methodological framework developed by Arksey and O'Malley ${ }^{36}$ and enhanced by Levac et al. ${ }^{37} \mathrm{~A}$ scoping review is suitable for this topic area as it is defined as 'a form of knowledge synthesis that addresses an exploratory research question aimed at mapping key concepts, types of evidence, and gaps in research related to a defined area or field by systematically searching, selecting and synthesizing knowledge. ${ }^{, 38}$ This method includes five stages: (1) identifying the research question; (2) identifying relevant studies; (3) study selection; (4) charting the data; and (5) collating, summarising and reporting the results. The aim of this review is for it to serve as a report that can guide 
the development of best practices for families in addiction treatment.

\section{Stage 1: identifying the research question}

Scoping reviews comprise broad research questions as their aim is to synthesise the breadth of evidence on a clearly identified area of inquiry. ${ }^{37}$ Through consultation with our research team, we developed one broad research question: What are the family interventions or practices that have been implemented and examined in adult addiction treatment? Levac $e t a l^{37}$ recommend that researchers define study concepts and target populations in scoping reviews. For this review, we define family broadly to include kin and significant others and this term also includes diverse family members or relationships including, but not limited to, partners, spouses, siblings, friends, adult children and parents. The term addiction encompasses any problematic substance use (alcohol or drugs), as well as problem gambling. Interventions or practices include any method of involving family members in addiction treatment that has been implemented and empirically examined. Treatment refers to any services for an adult coping with an addiction, problematic substance use or problem gambling.

\section{Stage 2: identifying relevant studies}

The comprehensive search strategy was developed in collaboration with the study's principal and coinvestigators (TK and RA), a health sciences librarian at the University of Toronto and the research assistants who will help conduct the scoping review. Relevant studies will be identified by searching the following electronic databases: MEDLINE, PsycINFO and Social Services Abstracts. The researchers selected a cross section of databases that include the range of literature spanning healthcare, psychology, psychiatry, social work and other social sciences. An initial scan of several databases demonstrated that the databases selected were more likely to identify results that are related to the focus of this scoping review. Other databases were not included in this study because the initial scan resulted in thousands of results that were not relevant to the scope of this study. However, to ensure that the scoping review captures the breadth of literature, the research team will also extensively handsearch various reference lists of included studies, key journals, as well as web sites of addiction-related organisations including the Canadian Centre on Substance Use and Addiction (CCSA), Gambling Research Exchange Ontario (GREO), Addiction and the Family International Network (AFINet), and Substance Abuse and Mental Health Services Administration (SAMHSA). The search will cover 2000 up to the present and this limited date range was selected as we are interested in recent addiction treatment approaches involving families in order for this to be most relevant for current treatment programmes. A preliminary search conducted by the research assistant and reviewed by the primary investigator helped refine the search terms and protocol. All literature searches will

\begin{tabular}{|c|c|}
\hline Concept & Search terms \\
\hline Family & $\begin{array}{l}\text { famil* OR significant other* OR conjoin* OR } \\
\text { sibling* OR spous* OR couple* OR partner* }\end{array}$ \\
\hline Addiction & $\begin{array}{l}\text { addict* OR abus* OR problem* OR misuse OR } \\
\text { use* adj2 } \\
\text { alcohol OR drug* OR substance OR gambl* }\end{array}$ \\
\hline Practice & 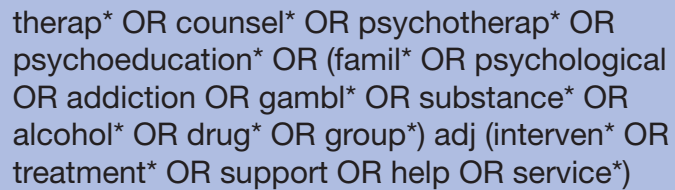 \\
\hline
\end{tabular}

be completed by a research assistant who will be supervised and guided by the primary investigator (TK). The search includes several terms that relate to these concepts: family, addiction and practice (see table 1).

\section{Stage 3: study selection}

We are following recommendations made by Levac et $a l^{37}$ and will use a transparent, iterative team approach to refine the search strategy and this will be an iterative process to finalise inclusion and exclusion criteria. The review process consists of two stages: first by screening of titles and abstracts and, second, by fulltext screening. A research assistant will review using the selection criteria and will be supervised closely by the lead researcher. The research assistant will mark the selections under review with 'include,' 'exclude' or 'uncertain'. Levac et $a l^{37}$ noted that it is important to clarify the process of decision-making and we have determined that uncertainties will be discussed with the lead researcher and a third investigator will be included when we cannot reach resolution. The following inclusion criteria will be used to guide the search and will also be used when reviewing articles: (1) study focuses on any type of family involvement or intervention in addiction treatment for family members who have a significant other coping with an addiction; (2) addiction includes alcohol, drugs and gambling at any level of severity; (3) published or unpublished empirical studies that use quantitative, qualitative or mixed methods; (4) written in English or French; and (5) from 2000 onwards. Exclusion criteria that have been identified are: (1) studies that focus on addictions in children or adolescents $<18$ years of age; (2) literature reviews; and (3) empirical studies that focus on the impact of addiction on family members, but do not examine involvement of family members in treatment.

\section{Stage 4: charting the data}

Our data will be charted and sorted according to key themes using a qualitative thematic analysis approach. ${ }^{39} 40$ This is a commonly used method for scoping reviews and it involves identifying themes across the literature and synthesising using summary 
tables with thematic headings. ${ }^{39}{ }^{40}$ Charting will be conducted by the research assistant and reviewed by the lead researcher. We will chart extracted data using a data charting form on Excel. Following the recommendation of Daudt et al, ${ }^{41}$ we will assign each paper an identifying number which will minimise errors and facilitate tracking of included and excluded articles. We also followed the recommendations by Levac et $a l^{37}$ to make charting an iterative process. The research assistant did a preliminary extraction of 10 articles with some preliminary categories for the chart and the charting was reviewed by the lead researcher. The research team discussed the themes and variables and updated the charting form. The themes and variables that we will be charting include: author, title, peer-reviewed or grey literature paper, country where study was conducted, research aims, methods, sample size, individual with addiction (if specified), family member (if specified), definition of family and family involvement (if provided), addiction type, intervention type, harm reduction or abstinence approach (if specified) and key findings of the study. For key findings, we are specifically searching for a study's results on family involvement in addiction treatment.

\section{Stage 5: collating, summarising and reporting the results}

Levac et $a l^{37}$ suggest that this stage is divided in three distinct steps. With this in mind, our research team will follow these steps in the final stage of the scoping review: (1) analyse extracted data using a numerical summary analysis and qualitative thematic analysis; (2) disseminate the results of the scoping review; and (3) discuss implications for future research, policy and practice.

\section{ETHICS AND DISSEMINATION}

This paper presents the protocol for a scoping review of family practices in addiction treatment. Ethics approval is not necessary as the data are collected from publicly available sources. This review will advance knowledge on the ways family members are involved in treatment when a significant other has a substance use or gambling problem. The results will be disseminated through a peer-reviewed journal and will also be reported at local, national and international conferences on addictions and mental health.

Contributors TK contributed to the project idea and both TK and RA provided guidance to the research assistant, and design of the search strategy. TK contributed to the literature review and RA and TK contributed to the writing and editing of the protocol. Both authors have made substantive intellectual contributions to the development of this protocol. Both read and approved the manuscript.

Funding This research received no specific grant from any funding agency in the public, commercial or not-for-profit sectors.

Competing interests None declared.

Provenance and peer review Not commissioned; externally peer reviewed.
Open Access This is an Open Access article distributed in accordance with the Creative Commons Attribution Non Commercial (CC BY-NC 4.0) license, which permits others to distribute, remix, adapt, build upon this work non-commercially, and license their derivative works on different terms, provided the original work is properly cited and the use is non-commercial. See: http://creativecommons.org/ licenses/by-nc/4.0/

(c) Article author(s) (or their employer(s) unless otherwise stated in the text of the article) 2018. All rights reserved. No commercial use is permitted unless otherwise expressly granted.

\section{REFERENCES}

1. United Nations Office on Drugs and Crime. World drug report 2017. United Nations: United Nations Office on Drugs and Crime, 2017.

2. World Health Organization. Substance abuse dept. global status report: alcohol policy. Geneva: WHO, 2004.

3. Calado F, Griffiths MD. Problem gambling worldwide: an update and systematic review of empirical research (2000-2015). J Behav Addict 2016;5:592-613.

4. Rehm J. The risks associated with alcohol use and alcoholism. Alcohol Res Health 2011;34:135.

5. Ray GT, Mertens JR, Weisner C. Family members of people with alcohol or drug dependence: health problems and medical cost compared to family members of people with diabetes and asthma. Addiction 2009;104:203-14.

6. Weisner C, Parthasarathy S, Moore C, et al. Individuals receiving addiction treatment: are medical costs of their family members reduced? Addiction 2010;105:1226-34.

7. Ahern J, Stuber J, Galea S, et al. Stigma, discrimination and the health of illicit drug users. Drug Alcohol Depend 2007;88:188-96.

8. Canadian Centre on Substance abuse. Substance abuse in Canada: concurrent disorders. Canada: Canadian Centre on Substance Abuse, 2009.

9. Borges G, Loera CR. Alcohol and drug use in suicidal behaviour. Curr Opin Psychiatry 2010;23:195-204.

10. Darvishi N, Farhadi M, Haghtalab T, et al. Alcohol-related risk of suicidal ideation, suicide attempt, and completed suicide: a metaanalysis. PLoS One 2015;10:e0126870.

11. Schneider B. Substance use disorders and risk for completed suicide. Arch Suicide Res 2009;13:303-16.

12. Orford J, Velleman R, Natera G, et al. Addiction in the family is a major but neglected contributor to the global burden of adult illhealth. Soc Sci Med 2013;78:70-7.

13. Copello A, Templeton L, Orford J, et al. The relative efficacy of two levels of a primary care intervention for family members affected by the addiction problem of a close relative: a randomized trial. Addiction 2009;104:49-58.

14. Copello AG, Templeton L, Velleman R. Family interventions for drug and alcohol misuse: is there a best practice? Curr Opin Psychiatry 2006;19:271-6.

15. Kourgiantakis T, Saint-Jacques MC, Tremblay J. Facilitators and barriers to family involvement in problem gambling treatment. Int $J$ Ment Health Addict 2017:1-22.

16. Csiernik R. Counseling for the family: the neglected aspect of addiction treatment in Canada. $J$ Soc Work Pract Addict 2002;2:79-92.

17. Copello A, Orford J. Addiction and the family: is it time for services to take notice of the evidence? Addiction 2002;97:1361-3.

18. Lee CE, Christie MM, Copello A, et al. Barriers and enablers to implementation of family-based work in alcohol services: A qualitative study of alcohol worker perceptions. Drugs 2012;19:244-52.

19. Copello AG, Velleman RD, Templeton LJ. Family interventions in the treatment of alcohol and drug problems. Drug Alcohol Rev 2005;24:369-85.

20. Edwards ME, Steinglass $P$. Family therapy treatment outcomes for alcoholism. J Marital Fam Ther 1995;21:475-509.

21. Epstein EE, McCrady BS. Behavioral couples treatment of alcohol and drug use disorders: current status and innovations. Clin Psychol Rev 1998;18:689-711.

22. Meads C, Ting S, Dretzke J, et al. A systematic review of the clinical and cost-effectiveness of psychological therapy involving family and friends in alcohol misuse or dependence. Health Technol Assess Rep 2007;65:97.

23. Hasin D, Keyes K. The epidemiology of alcohol and drug disorders. In: Johnson BA, ed. Addiction medicine. New York: Springer, 2010:23-49. 
24. Suurvali $H$, Hodgins D, Toneatto $T$, et al. Treatment seeking among Ontario problem gamblers: results of a population survey. Psychiatr Serv 2008;59:1343-6.

25. Brorson $\mathrm{HH}$, Ajo Arnevik E, Rand-Hendriksen K, et al. Drop-out from addiction treatment: a systematic review of risk factors. Clin Psychol Rev 2013;33:1010-24.

26. Ladouceur R, Gosselin P, Laberge M, et al. Dropouts in clinical research: do results reported reflect clinical reality? Behav Ther 2001;24:44-6.

27. Bischof G, Iwen J, Freyer-Adam J, et al. Efficacy of the community reinforcement and family training for concerned significant others of treatment-refusing individuals with alcohol dependence: A randomized controlled trial. Drug Alcohol Depend 2016;163:179-85

28. Hodgins DC, Toneatto T, Makarchuk K, et al. Minimal treatment approaches for concerned significant others of problem gamblers: a randomized controlled trial. J Gamb/ Stud 2007;23:215-30.

29. Roozen $H G$, de Waart $R$, van der Kroft P. Community reinforcement and family training: an effective option to engage treatmentresistant substance-abusing individuals in treatment. Addiction 2010;105:1729-38.

30. McCrady BS, Epstein EE. Overcoming alcohol problems: a couplesfocused program workbook. New York: Oxford University Press, 2009.

31. Orford J, Copello A, Velleman R, et al. Family members affected by a close relative's addiction: the stress-strain-coping-support model. Drug 2010;17(suppl 1):36-43.
32. Lucksted A, McFarlane W, Downing D, et al. Recent developments in family psychoeducation as an evidence-based practice. J Marital Fam Ther 2012;38:101-21.

33. Orford J, Natera G, Copello A, et al. Coping with alcohol and drug problems: the experiences of family members in three contrasting cultures. London: Taylor \& Francis, 2005.

34. Hampson CL. Integrating family-focused practice into routine addiction services. Doctoral dissertation, University of Birmingham, 2012.

35. American Psychiatric Association. Diagnostic and statistical manual of mental disorders (DSM-5®). American Psychiatric Pub, 2013.

36. Arksey H, O'Malley L. Scoping studies: towards a methodological framework. Int J Soc Res Methodol 2005;8:19-32.

37. Levac D, Colquhoun H, O'Brien KK, et al. Scoping studies: advancing the methodology. Implement Sci 2010;5:69.

38. Colquhoun HL, Levac D, O'Brien KK, et al. Scoping reviews: time for clarity in definition, methods, and reporting. J Clin Epidemiol 2014;67:1291-4.

39. The Joanna Briggs Institute. Joanna briggs institute reviewers' manual: 2015 edition / supplement: JBI Database System Rev Implement Rep.

40. Kastner M, Tricco AC, Soobiah C, et al. What is the most appropriate knowledge synthesis method to conduct a review? Protocol for a scoping review. BMC Med Res Methodol 2012;12:114.

41. Daudt HM, van Mossel C, Scott SJ. Enhancing the scoping study methodology: a large, inter-professional team's experience with Arksey and O'Malley's framework. BMC Med Res Methodol 2013;13:48 